\title{
THE TODA FLOW ON A GENERIC ORBIT IS INTEGRABLE
}

\author{
BY P. DEIFT, L. C. LI, T. NANDA AND C. TOMEI
}

\begin{abstract}
For the generic orbit of the coadjoint action of the lower triangular group on its dual Lie algebra, we exhibit a complete set of integrals in involution for the associated Toda flow.
\end{abstract}

The Toda flow $[\mathbf{1}-\mathbf{3}]$ on an orbit of the coadjoint action of the identity component $L$ of the lower triangular group on its dual Lie algebra $\{S: S=$ $\left.S^{T}\right\}$ is generated by the Hamiltonian $\frac{1}{2} \operatorname{tr} S^{2}$. As is well known [1-5], the eigenvalues of $S$ provide integrals in involution for the flow. In particular, the flow on an orbit consisting of tridiagonal matrices is completely integrable. The purpose of this note is to describe sufficient additional integrals to prove that the Toda flow on a generic coadjoint orbit is also integrable.

For an $n \times n$ matrix $M$, define the polynomial

$$
\begin{aligned}
P_{k}(M, \lambda) & \equiv \operatorname{det}\left\{(M-\lambda)_{i j}: k+1 \leq i \leq n, 1 \leq j \leq n-k\right\}, \\
& =\sum_{r=0}^{n-2 k} E_{r, k}(M) \lambda^{n-2 k-r}, \quad 0 \leq k \leq\left[\frac{n}{2}\right] .
\end{aligned}
$$

The signs of $E_{0, k}(S)$ are preserved under the coadjoint action of $L$. We say that an orbit $O_{S}$ through $S$ is generic if $E_{0, k}(S)$ is nonzero, $0 \leq k \leq[n / 2]$. On a generic orbit define

$$
I_{r, k}(S)=E_{r, k}(S) / E_{0, k}(S), \quad 0 \leq k \leq[(n-1) / 2], 1 \leq r \leq n-2 k .
$$

THEOREM 1. The generic orbit $O_{S}$ is the set $\left\{U=U^{T}: \operatorname{sgn} E_{0, k}(U)=\right.$ $\left.\operatorname{sgn} E_{0, k}(S), 0 \leq k \leq[n / 2], I_{1, k}(U)=I_{1, k}(S), 0 \leq k \leq[(n-1) / 2]\right\}$ and has dimension $2\left[n^{2} / 4\right]$. The functions $I_{r, k}(S), 0 \leq k \leq[(n-1) / 2], 2 \leq r \leq n-2 k$, provide $\left[n^{2} / 4\right]$ integrals in involution for the Toda flow. Furthermore, the integrals are independent on a dense open set in $O_{S}$.

Let $\mathrm{GL}_{k}(n), 0 \leq k \leq[(n-1) / 2]$, denote the identity component of the group of invertible matrices obtained by setting equal to zero all entries which are strictly above the main diagonal and lie in the first $k$ rows or the last $k$ columns. A key fact in the proof of Theorem 1 is that each $I_{r, k}$ is invariant under the restriction to $\mathrm{GL}_{k}(n)$ of the coadjoint action of $\mathrm{GL}(n)$ on its dual Lie algebra, i.e., $I_{r, k}\left(g^{T} M\left(g^{T}\right)^{-1}\right)=I_{r, k}(M)$ for all $g \in \mathrm{GL}_{k}(n)$ and all matrices $M$. In particular, as $L \subset \mathrm{GL}_{k}(n)$, it follows as in the Kostant-Symes theorem (see e.g. [3]) that the Poisson brackets for the $I_{r, k}$ 's on $O_{S}$ can be computed in $T^{*}(\mathrm{GL}(n))$, where the invariance under the full group $\mathrm{GL}_{k}(n)$ is then used to prove involution. Also the flows on the orbit $O_{S}$ are projections of the associated flows on $T^{*}(\mathrm{GL}(n))$. Indeed, we have

Received by the editors March 2, 1984 and, in revised form, April 18, 1984.

1980 Mathematics Subject Classification. Primary 22E25, 58F07, 70 H99.

(C) 1984 American Mathematical Society $0273-0979 / 84 \$ 1.00+\$ .25$ per page 
THEOREM 2 (CF. [3]). For any $r, k$, let $(Q(t), P(t)),(Q(0), P(0))=$ $(1, S)$, be the (global) flow induced on $T^{*}(\mathrm{GL}(n))=\{(Q, P): Q \in \mathrm{GL}(n)$; $P$ any $n \times n$ matrix $\}$ by the Hamiltonian $\tilde{I}_{r, k}(Q, P) \equiv I_{r, k}\left(Q^{T} P\right)$. Then

$$
S(t)=Z(t)^{T} S Z(t)
$$

solves the $I_{r, k}$ flow on $O_{S}$ with initial condition $S(0)=S$, where $Q(t)=$ $Z(t) X(t)$ is the $Q L$-decomposition of $Q(t)$.

The flows generated on $O_{S}$ by the Hamiltonians $I_{r, 0}$, the symmetric functions, always converge as $t \rightarrow \pm \infty[6]$. However, the flows generated by $I_{r, k}, k \geq 1$, can have periodic orbits. Thus, in contrast to the tridiagonal case, where the invariant sets for the Toda flow are products of lines (see e.g. [5-7]), in the generic case the invariant sets are products of lines and circles.

The $E_{r, k}$ 's defined above can also be used to prove integrability for the Toda flow on (generic) orbits of band matrices.

Finally, from a different point of view, it is possible to decompose the Toda flow on general orbits into a union of independent Toda flows on traditional (and hence integrable) orbits.

ACKNOWLEDGEMENTS The work of the first author was supported in part under NSF Grant \# MCS-8301662 and ONR Grant \# NO0014-76-C-0439, and that of the last author under NSF Grant \# MCS-8300568. The work of the third author was supported in part by the Office of Energy Research under contract W-7404-ENG-48. The last author would like to thank N. Wildberger for many useful conversations.

\section{REFERENCES}

1. M. Adler, Invent. Math. 50 (1979), 219.

2. B. Kostant, Adv. in Math. 34 (1979), 195.

3. W. W. Symes, Phys. D 1 (1980), 339.

4. H. Flaschka, Phys. Rev. B 9 (1974), 1924.

5. J. Moser, Lecture Notes in Phys. 38 (1975), 467.

6. P. Deift, L. C. Li and C. Tomei, Toda flows with infinitely many variables (to appear).

7. P. Deift, T. Nanda and C. Tomei, SIAM J. Numer. Anal. 20 (1983), 1. 10012

Courant Institute of Mathematical Sciences, New York, New York

DEPARTMENT OF MATHEMATICS, NATIONAL UNIVERSity OF Singapore, Kent RIDGE, SINGAPORE 0511

Department OF MATHEMATICS, UNIVERsity OF CAlifornia, Berkeley, CALIFORNIA 94720

Department of Mathematics, Yale University, New Haven, ConnectiCUT 06520 